\title{
SCIENTIFIC REPORTS

\section{OPEN In-depth proteome analysis of more than 12,500 proteins in buffalo mammary epithelial cell line identifies protein signatures for active proliferation and lactation}

\author{
Shalini Jaswal ${ }^{1}$, Vijay Anand ${ }^{2}$, Sudarshan Kumar ${ }^{1}$, Shveta Bathla ${ }^{1}$, Ajay K. Dang ${ }^{3}$, \\ Jai K. Kaushik ${ }^{1} \&$ Ashok K. Mohanty $^{1 *}$
}

The mature mammary gland is made up of a network of ducts that terminates in alveoli. The innermost layer of alveoli is surrounded by the differentiated mammary epithelial cells (MECs), which are responsible for milk synthesis and secretion during lactation. However, the MECs are in a state of active proliferation during pregnancy, when they give rise to network like structures in the mammary gland. Buffalo (Bubalus bubalis) constitute a major source of milk for human consumption, and the MECs are the major precursor cells which are mainly responsible for their lactation potential. The proteome of MECs defines their functional state and suggests their role in various cellular activities such as proliferation and lactation. To date, the proteome profile of MECs from buffalo origin is not available. In the present study, we have profiled in-depth proteome of in vitro cultured buffalo MECs (BUMECs) during active proliferation using high throughput tandem mass spectrometry (MS). MS analysis identified a total of $8330,5970,5289,4818$ proteins in four sub-cellular fractions (SCFs) that included cytosolic (SCF-I), membranous and membranous organelle's (SCF-II), nuclear (SCF-III), and cytoskeletal (SCF-IV). However, 792 proteins were identified in the conditioned media, which represented the secretome. Altogether, combined analysis of all the five fractions (SCFs-I to IV, and secretome) revealed a total of 12,609 non-redundant proteins. The KEGG analysis suggested that these proteins were associated with 325 molecular pathways. Some of the highly enriched molecular pathways observed were metabolic, MAPK, PI3-AKT, insulin, estrogen, and cGMP-PKG signalling pathway. The newly identified proteins in this study are reported to be involved in NOTCH signalling, transport and secretion processes.

The mammary gland, a unique organ of mammals, is a derivative of ventral skin ${ }^{1}$ and has evolved to provide nutrition and immune protection to the offspring. Though the anatomical and physiological features of a mammary gland are diverse, the basic structure is similar in all species. The mature mammary gland is constituted by a network of tubular-alveolar structures, with their lumen lined by a layer of polarized secretory mammary epithelial cells (MECs). The MECs are dynamic components of the mammary gland, which undergo repeated rounds of proliferation and differentiation during different developmental stages such as pregnancy, and lactation ${ }^{2}$. The quantity and functional differentiation of MECs in the udder determine the milk-producing ability in livestock. Bovine (ruminants) are physiologically distinct from other mammals such as humans, mice and rats. The present study on in-depth proteome analysis of buffalo (Bubalus bubalis) MECs (BuMECs) advances our knowledge on the protein machinery operating in mammary gland development and lactogenesis in buffalo, which can be extrapolated in other mammalian species as well. Among the domestic livestock species, cattle (Bos taurus) and

${ }^{1}$ Proteomics and Structural Biology Lab, Animal Biotechnology Center, National Dairy Research Institute, Karnal, 132001, Haryana, India. ${ }^{2}$ Department of Veterinary Physiology and Biochemistry, Veterinary College and Research Institute (TANUVAS), Orathanadu, 614625, Tamilnadu, India. ${ }^{3}$ Dairy Cattle Physiology Division, National Dairy Research Institute, Karnal, 132001, Haryana, India. *email: ashokmohanty1@gmail.com 
buffalo are the major contributors to the production of nutritious milk for human consumption. India harbours the largest number (57\%) of buffaloes in the world that contribute around $51.2 \%$ of milk produced in India and $13 \%$ of total milk supply in world ${ }^{3}$. Buffalo is the second-largest milk-producing animal, and its milk is rich in fat, solids- not-fat (SNF) and protein content compared to that of cow's milk ${ }^{4}$. Buffaloes also play an essential role in the development of the economy for the farming community by providing milk, meat, and draft power.

MEC lines from different species serve as a model system to gain insight into the biological functions of the mammary gland. In the present study, we have profiled and analyzed the in-depth proteome of BuMEC line ${ }^{5}$ developed in our lab using a high throughput proteomics approach. Previous studies on global proteome profiling of MECs isolated from milk of cattle ${ }^{6}$ and human (MCF-7) 7 reported a total of 497 and 3,715 proteins, respectively. Also, various other groups have reported combined proteome from multiple biological sources such as 10,361 \pm 120 proteins, each in 11 different human cell lines ${ }^{8}, 10,350$ in NCI- $60^{9}$, and 7,349 in 28 mice tissues $^{10}$. Although the above number of proteins represents a sizeable proteome coverage, recent advancements in the mass spectrometry (MS) have enabled the analysis of complex cellular proteins in high numbers ${ }^{8}$. The main challenge in any proteomic study is to identify the cellular proteins available in minor quantities, which are usually masked by the abundant ones during MS analysis. Identification of proteins from different sub-cellular organelles followed by peptide fractionation and MS analysis is an advanced method for in-depth proteome analysis ${ }^{11}$.

In the present study, we performed in-depth proteome profiling of actively proliferating BuMECs. Ruminants (cattle, buffalo, goat, etc.) constitute a distinct class of livestock species, which are known milk producers. Their physiology is different from other non- ruminant mammalian species such as human, mice, monkey, rat etc. Given the differences in mammary gland physiology between ruminants and non-ruminants, expression of various proteins and molecular pathways associated with proliferation and lactogenesis, mimicking in vivo mammary gland development and lactation respectively, may be different in BuMECs. To our knowledge, no information is available on the proteome of BuMECs. Moreover, we have performed proteome analysis of 68 digested protein fractions [24 digested protein fractions for sub-cellular fraction-I (SCF-I); 12 digested protein fractions- each for SCF-II, -III, and -IV; and 8 digested protein fractions for conditioned media] to generate in-depth proteome data, which is the uniqueness of this study. Hence, the present study provides new insights into the molecular physiology of mammary gland associated with its development and lactation.

The primary objective was to identify the maximum number of proteins by adopting advanced methodologies that included sub-cellular protein fractionation followed by peptide fractionation and high-resolution MS. The MS analysis of four SCFs and conditioned media helped us to identify the highest number of proteins reported so far in any mammalian cell line. Furthermore, the Bioinformatics analysis mapped the proteins to various molecular pathways, which may serve as the key regulators for the active proliferation of BuMECs. The current dataset on BuMECs proteome will add to the existing information available on mammary proteome and, constitute a reservoir of proteins for further investigation and characterization of the ruminant mammary system in general, particularly the buffalo.

\section{Results}

Shotgun proteome analysis of sub-cellular protein fractions and conditioned media. The four SCFs which included cytosolic (SCF-I); membranous and membranous organelles (SCF-II); nuclear (SCF-III); and cytoskeletal (SCF-IV)], and conditioned media, from actively proliferating BuMECs were analyzed for identification of proteins using the MS. Data were generated and analyzed separately for each of the five fractions and further combined for the generation of comprehensive proteome profile of actively proliferating BuMECs. A total of 8330 (Table S1), 5970 (Table S2), 5289 (Table S3) and 4818 (Table S4) non-redundant proteins were identified in SCFs (I-IV) respectively with $1 \%$ false discovery rate (FDR). The MS analysis of conditioned media identified a total of 792 non-redundant proteins (Table S5), which represent the secretome of actively proliferating BuMECs. Of these proteins 195 were identified with $\geq 2$ peptides. Analysis of 195 proteins using SignalP software suggested that a total of 35 proteins contained a typical signal peptide sequence. The presence of signal peptide sequence demonstrated their secretory nature (Table S6). Altogether, combined analysis of proteins from all the five fractions (SCFs- I to IV, and conditioned media) revealed a total of 12,609 non-redundant proteins in actively proliferating BuMECs (Table S7).

Gene Ontology (GO), Protein-Protein Interaction (PPI) and Pathway analysis of identified proteins. Out of total proteome obtained, 10,173 (80.7\%) proteins with $\geq 2$ unique peptides were subjected to further Bioinformatics analysis. These proteins represented approximately $63.0 \%$ of the protein-coding sequences (CDSs) predicted from the Bos taurus genome. GO analysis using Protein Analysis Through Evolutionary Relationships (PANTHER) ${ }^{12}$ categorized the proteins into the biological process (BP), molecular function (MF) and cellular component (CC). The top three GO terms for BP were the cellular process $(32.2 \%)$, metabolic process (22.1\%) and biological regulation (14.8\%). In the MF category, the identified proteins were mainly involved in binding (36.5\%) and catalytic (35.5\%) functions. The GO-based on CC mapped $42.6 \%$ and $30.2 \%$ of the identified proteins to the cell and organelle, respectively (Fig. 1). The Kyoto Encyclopedia of Genes and Genomes (KEGG) analysis of the proteins was performed to identify the signalling pathways associated with the active proliferation of BuMECs. The results suggested that the proteins were involved in 325 signalling pathways. The pathways such as metabolic, MAPK, RAP1, mTOR, insulin, oxytocin, AMPK, and JAK-STAT with protein counts of 790, 197, $139,114,112,111,93$, and 78 respectively, revealed the protein signatures associated with active proliferation of BuMECs (Table S8). Since a large number of proteins (790) were found to be involved in the metabolic pathways, their roles in metabolism were investigated further using Metscape (Cytoscape Plug-in) ${ }^{13}$. This resulted in the generation of a network based on the known PPIs and Protein-Metabolite Interactions (PMIs) (Fig. 2a). Metscape analysis suggested that the proteins were associated with 92 metabolic pathways (Table S9). Notable pathways included de novo synthesis of fatty acids (Fig. 2b-I); $\beta$-oxidation of fatty acids (Fig. 2b-II); biosynthesis and 


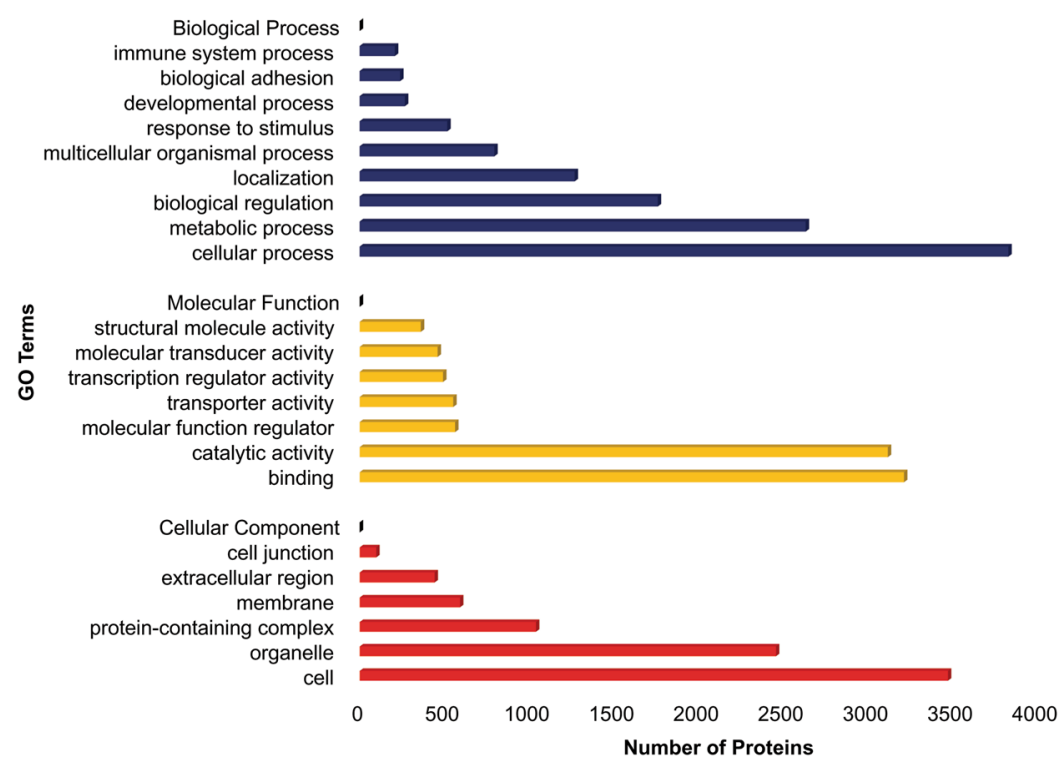

Figure 1. Gene Ontology (GO) classification of the proteins associated with the active proliferation of buffalo mammary epithelial cells (BuMECs). GO analysis divided proteins into three functional groups: Biological Process (BP), Molecular Function (MF) and Cellular Component (CC). Regarding BPs, proteins were mainly involved in cellular and metabolic activities. In terms of the MFs, the major activities associated with proteins were binding and catalytic. The majority of the proteins were present in cell parts, organelles and proteincontaining complex.

metabolism of androgen, estrogen, C-21 steroid hormones, glycosphingolipids (ganglio-, globo- and lacto-series), cholesterol, vitamin B5, glycans (N- and O-) and prostaglandin; glycolysis and gluconeogenesis (Fig. 2b-III); pentose phosphate pathway (Fig. 2b-IV); metabolism of amino acids (biopterin, tryptophan, and tyrosine), vitamins (A, B, D3, E, $\mathrm{H}$, and $\mathrm{K}$ ) and purine.

The PPIs mediates complex cellular processes ${ }^{14}$. The significance of the PPI network built by the identified proteins was investigated using Search Tool for the Retrieval of Interacting Genes/Proteins (STRING) ${ }^{15}$ version 11.0 (https://string-db.org), and Cytoscape software version 3.6.1 (https://www.cytoscape.org). To achieve this, top 2000 proteins based on peptide number were mapped to the STRING database, which results in the generation of PPI network. From this network PPIs with a high confidence score of $\geq 0.9$ were screened. These interactions were further used to build a PPI network using Cytoscape. The PPI network generated using Cytoscape consisted of 1889 nodes and 8813 interactions (Fig. 3a). The Molecular Complex Detection (MCODE) ${ }^{16}$ analysis of the PPI network, then resulted in the identification of 138 protein modules, also known as clusters (Table S10). Of the 138 modules, the top three modules with the highest scores are represented in Fig. 3b. Module 1 (I) with a score of 44.364, consisted of 45 nodes and 976 edges (Fig. 3b-I). Module 2 (II) with a score of 38, consisted of 38 nodes and 703 edges (Fig. 3b-II). Whereas, Module 3 (III) with a score of 32.606, consisted of 34 nodes and 538 edges (Fig. 3b-III). The pathway enrichment analysis demonstrated that the proteins in module I were enriched with various biological functions such as mitochondrial translation and PPAR-alpha pathway, whereas the proteins in other two modules (-II and -III) were associated with intra-Golgi and retrograde Golgi-to-ER traffic, aurora B signalling, translocation of GLUT4 to the plasma membrane, PLK1 signalling, insulin processing, Class I MHC mediated antigen processing and presentation, and ubiquitin-mediated proteolysis (Table S11).

In the present study, GO analysis of the total identified proteins suggested that the $36.5 \%$ protein were associated with the binding functions. The DNA binding proteins regulate the expression of genes during growth and differentiation of the cells ${ }^{17}$. To identify the DNA binding proteins or transcription factors (TFs), we compared the present proteome dataset with the Animal transcription factor database (Animal TFDB) of Bos taurus origin (http://bioinfo.life.hust.edu.cn/AnimalTFDB/). The results suggested that a total of 583 proteins were TFs, 261 were transcription-co factors (TCFs) and 102 were chromatin remodelling factors (CRFs) (Table S12). Altogether, 946 proteins were found to have DNA binding functions. The Database for Annotation, Visualization and Integrated Discovery (DAVID) ${ }^{18}$ analysis of DNA binding proteins suggested that most of them (51.6\%) were nuclear in origin (Table S13) and were enriched in the transcription process (23.3\%) (Table S14).

GO enrichment, PPI network and Pathway analysis of unique proteins from the SCFs and secretome. The proteins ( $\geq 2$ peptides) unique to sub-cellular compartments (SCF-I to IV) and secretome were identified by comparing proteome datasets obtained for the five fractions. The Venn diagram was constructed using the JVENN tool to display the common and unique proteins among five fractions diagrammatically ${ }^{19}$. The results suggested that $2449,665,523,480$ and 12 proteins were unique to the SCF- I, -II, -III, -IV, and secretome respectively (Fig. 4). Whereas, a total of 85 proteins were common among the five fractions (Table S15). Further, DAVID analysis suggested the association of cytosol specific proteins to metabolic, MAPK and PI3-AKT signalling pathways (Table S16); membrane specific proteins to TCA cycle, fatty acid metabolism, and PPAR 

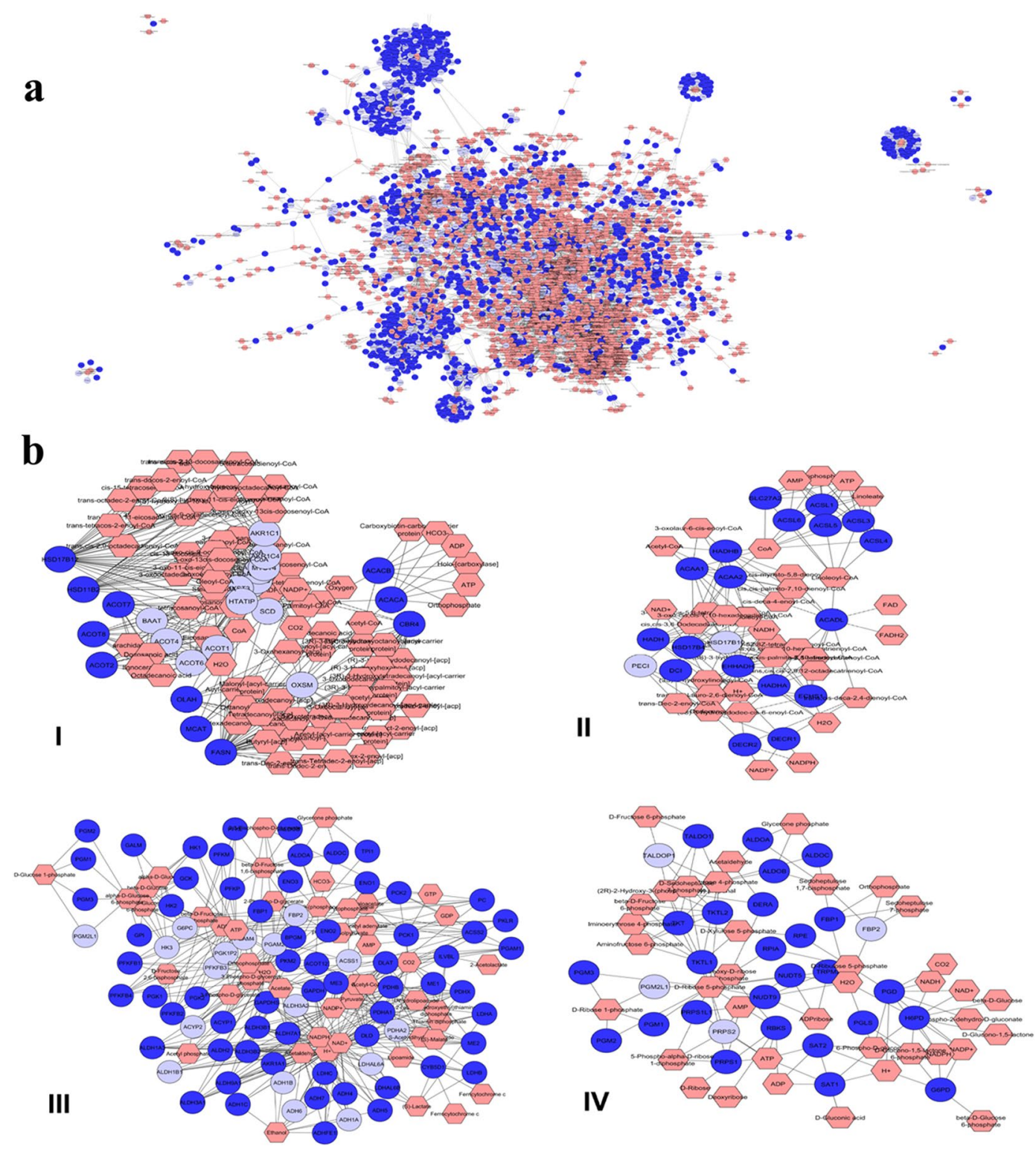

Figure 2. Correlation analysis of proteins and metabolites regulated in actively proliferating buffalo mammary epithelial cells (BuMECs). (a) Metscape (Cytoscape Plug-in) analysis generated a complex network comprising input proteins (spherical nodes and dark blue), metabolite (hexagons nodes and neon pink), and reactions (edges). (b) Major metabolic pathways generated. A total of 92 metabolic pathways were associated with the proteins identified in actively proliferating BuMECs. Selected pathways, including de novo fatty acid biosynthesis (I), di-unsaturated fatty acid beta-oxidation (II), glycolysis and gluconeogenesis (III), and pentose phosphate pathway (IV) are shown as subnetworks.

pathway (Table S17); nuclear specific proteins to MAPK, spliceosome, estrogen signalling and osteoclast differentiation pathway (Table S18); and cytoskeletal specific proteins to ribosome biogenesis, regulation of actin cytoskeleton, axon guidance and SNARE interactions in vesicular transport (Table S19). 35 secretory proteins identified in the conditioned media were involved in the ECM-receptor interactions, focal adhesion, cancer, and TGF- $\beta$ signalling pathways (Table S20). The proteins common among five fractions were enriched with 23 KEGG pathways. The enriched functions of common proteins mapped mostly to focal adhesion, tight junction, metabolic pathways (Glycolysis/Gluconeogenesis), ECM-Receptor interaction and PI3K-AKT signalling pathway (Table S21).

\section{Discussion}

The proteome signature of an actively proliferating cell conveys in-depth information on the fundamental biological processes associated with its cellular growth and development. MECs are the precursors that are responsible for lactogenesis, and their number is an indicator of milk-producing ability in livestock ${ }^{20}$. Currently, there is very little information available on the proteome profile of MECs of buffalo origin among the livestock species. 

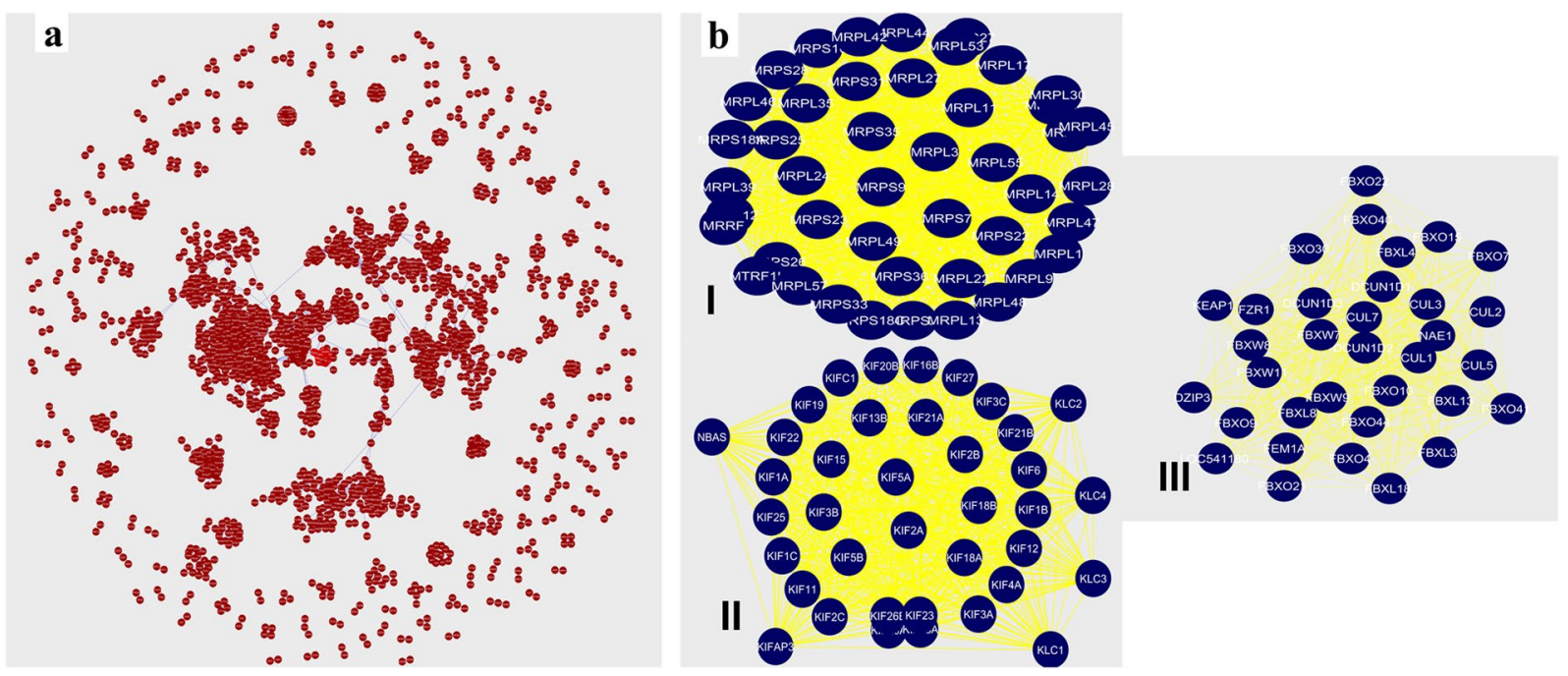

Figure 3. Protein-protein interaction (PPI) network analysis of top 2000 proteins (based on number of peptides identified for each protein) (a) The PPI network was constructed using the Cytoscape where each node represents a protein, and edges represent the interaction between two proteins. The PPI network consisted of 1889 nodes and 8813 edges. (b) Molecular Complex Detection (MCODE) analysis. A total of 138 clusters/ modules were extracted from the PPI network using Cytoscape plug-in MCODE. Top three modules with high scores representing Module 1 (I) (MCODE score $=44.364$, nodes $=45$ and edges $=976$ ) suggested the role of proteins in mitochondrial translation, Module $2($ II $)($ MCODE score $=38$, nodes $=38$ and edges $=703)$ consisted of proteins having role in intra-Golgi and retrograde Golgi-to-ER traffic, and Module 3 (III) $($ MCODE score $=32.606$, nodes $=34$ and edges $=538)$ with proteins mainly involved in class I MHC mediated antigen processing and presentation, and ubiquitin-mediated proteolysis.

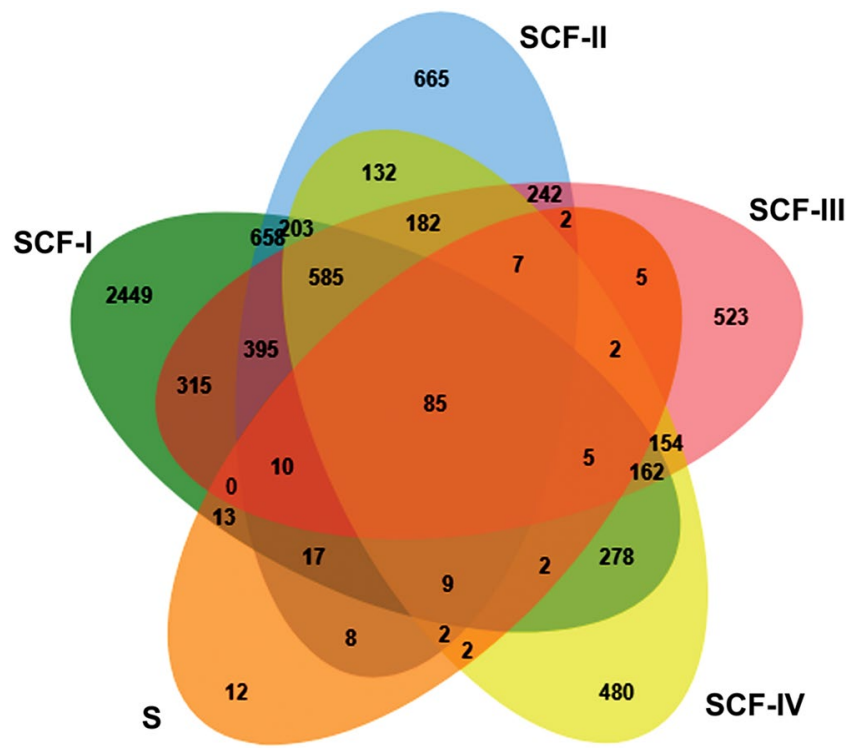

Figure 4. JVENN analysis. JVENN, an interactive Venn diagram viewer software, was used for the comparison of proteome datasets obtained from four sub-cellular protein fractions (SCFs I-IV) and secretome (S). The overlapping area represents proteins shared among the respective fractions. A total of 85 proteins were common among all the fractions.

In the present study, the use of sub-cellular protein fractionation and secretome isolation followed by a shotgun approach identified a total of 12,609 non- redundant proteins which is the most comprehensive proteome profile of any mammalian cell line. The data generated in this study will be useful to understand the mammary gland development in ruminants, particularly in buffalo. Also, the present study will further help to understand lactation biology as the number of MECs in the udder determine the lactation yield of an animal.

Few similar studies have been attempted in MECs from ruminants and other mammals. Jena et al. ${ }^{21}$, reported 43 differentially expressed proteins (DEPs) among tissues taken from developing and lactating mammary in 
buffalo. The Annexin A2 (ANXA2) and peroxiredoxin-6 (PRDX6) were found to be upregulated in Heifer mammary tissue. In contrast, eukaryotic translation elongation factor 1 delta transcript variant 2 (EEF1D), endoplasmic reticulum chaperone $\mathrm{BiP}$ (HSPA5), beta-lactoglobulin (LGB), and tissue-specific transplantation antigen P53B (TSTA3) were overexpressed in the lactating buffalo mammary tissue. Janjanam et al. ${ }^{22}$ suggested that AKT, $\mathrm{PI} 3 \mathrm{~K}$, and p38/MAPK signalling pathways were related to higher milk yield in cattle. Milk fat globule membrane (MFGM) secreted from the apical membrane carries a part of the cytoplasmic fraction of MECs, and they also represent partial information on the proteome of terminally differentiated MECs ${ }^{23}$. Studies on MFGM proteome reported a total of $632,137,947$ and 1104 proteins in cattle, goat, donkey, and human respectively ${ }^{24,25}$. On the other hand, a proteomics study on mice mammary tissue suggested the biological significance of RNA polymerase B transcription factor 3 (BTF3) in the differentiation of MECs during pregnancy ${ }^{26}$. Cytokeratin 15 (CK15) and dihydropyriminidase-related protein 3 (DRP3) were suggested as markers for the normal breast epithelial cells ${ }^{27}$.

The MECs undergoes proliferation and differentiation based on the physiological status of an animal. Several proteins reported in this study characterized the BuMECs in actively proliferating state. More precisely, the Estrogen receptor (ESR 1 and 2) and various TFs such as proto-oncogene FOS (FOS), cyclic AMP-dependent transcription factor-4 (ATF4), cyclic AMP-responsive element-binding protein 1 (CREB1), CREB3, CREB3L4, specificity protein 1 (SP1), Jun oncogene (JUN), activating transcription factor 2 (ATF2) and ATF6B, which are associated with its signalling were found in the proteome dataset of BuMECs. Estrogen stimulus in a developing mammary gland is important for parenchymal growth ${ }^{28,29}$ and branching morphogenesis ${ }^{30}$. The presence of ESR 1 and 2 in this study suggests that BuMECs have the potential to respond to Estrogen stimulus.

We report for the first time the presence of proteins such as actin-binding LIM (abLIM), secreted phosphoprotein 1 (SPP1), osteopontin (OPN), furry homolog (FRY), and FRY like transcriptional coactivator (FRYL) in BuMECs. abLIMs (isoforms 1-3) function as F-actin binding proteins, which mediates the formation of the actin filament network. They were reported to regulate gene expression, cell-matrix interactions, and cytoskeletal organization. These functions are essential for the cell division, and proliferation ${ }^{31}$. Human isoforms of OPN were reported to impart stemness in MECs ${ }^{32}$. FRY and its paralogue FRYL were suggested to act synergistically in the development and function of kidney ${ }^{33,34}$. The presence of these proteins in our dataset suggests that they may have a potential role during mammary gland morphogenesis in ruminants, which needs further investigation.

We also observed 30 different variants of cytokeratins (CKs) in the proteome of BuMECs. CKs are the major protein components of the intermediate filaments providing mechanical support to the epithelial cells $\mathrm{s}^{35}$. The expression pattern of CKs was used as a marker to characterize the MECs ${ }^{36}$. Various CKs such as 4, 5, 8, 23, 80 and 78 were reported to be the markers for progenitor cell ${ }^{37}$ and for non-invasive properties ${ }^{38,39}$. Their expression suggests the immortal nature of BuMECs with non-invasive characteristics.

We also report for the first time the presence of ion channel proteins such as Unc-79 homolog, NALC channel complex subunit (UNC79), Unc-80 homolog, NALC channel complex subunit (UNC80), sodium leak channel non-selective protein (NALCN) and aquaporin 6 (AQP6) in the BuMECs proteome dataset. Three proteins, including UNC79, UNC80 and NALCN, are reported to form a cation channel complex in the neurons and myometrium myocytes. The channel is permeable to $\mathrm{Na}^{+}, \mathrm{K}^{+}$and $\mathrm{Ca}^{+}$ions, by which it regulates the resting membrane potential and excitability in neurons ${ }^{40,41}$. AQP6 is one of the members of AQP family, which includes the transmembrane proteins mainly involved in water transport. However, AQP6 does not have a significant role in water transport. Instead, it exists as an anion channel and mediates the uptake of ions at acidic $\mathrm{pH}^{42}$. It is predominantly expressed in the kidney, but its detailed mechanism of action is not well defined ${ }^{43}$. A previous study did not detect AQP6 in bovine mammary gland ${ }^{44}$. Ion channels and aquaporins were reported to be involved in cell proliferation, cell cycle progression, and cell migration, in response to different stimuli ${ }^{45,46}$.

About 40 ATP-binding cassettes (ABC) were found in BuMECs proteome, which included ABCA (11), ABCB (8), ABCC (11), ABCD (3), ABCE (1), ABCF (3) and ABCG (3). A total of 48 ABCs are reported to date, which has been classified into seven families (A to $G$ ) based on their amino acid sequences and organization of ATP-binding domains ${ }^{47}$. The number as well as expression of ABCs varies among different tissues in human ${ }^{48}$. $\mathrm{ABCs}$ are involved in the transport of cholesterol and other lipids in the mammary gland during lactation ${ }^{49}$.

We identified few germ cell-specific proteins such as testis-specific protein Y-linked 2 (TSPY2), a disintegrin and metalloprotease domain-containing protein (ADAM), ATP-dependent RNA helicase VASA (VASA) and JY-1 in BuMECs proteome dataset. TSPY suppresses p53 mediated apoptotic pathway and enhances the proliferation of spermatogonial cells $s^{50}$. ADAMs are transmembrane proteins, which play an important role in the Notch signalling $^{51}$, and various biological activities such as proteolysis, adhesion, and migration ${ }^{52}$. Out of a total of 34 reported proteins belonging to ADAM group ${ }^{53,54}, 11$ members $(1,2,7,10,17,19,21,22,30,32$, and 33) were identified in BuMECs. VASA is a primordial germ cell (PGC) specific RNA helicase, which play important roles during spermatogenesis and oogenesi ${ }^{55}$. JY-1 is an oocyte-specific marker, which mediates the nuclear maturation and cumulus expansion in oocytes ${ }^{56}$. The presence of these proteins suggests a possible role in the active proliferation of BuMECs, which need further investigation.

In ruminants, the number and secretory activity of MECs determine the milk yield and persistency of lactation $^{57}$. The amount of MECs within the mammary gland depends on their proliferation. The proteome dataset of BuMECs contained a large number of proteins associated with phosphatidylinositol 3-kinase (PI3K)/protein kinase B (AKT) (225 Proteins) $)^{58}$, mTOR (114 Proteins) ${ }^{59}$ and nuclear factor-kappa $\beta$ (NFK- $\beta$ ) (50 Proteins) ${ }^{60}$ signalling pathways, which are reported to have a direct role in the proliferation of MECs. Synthesis and secretion of milk components are among the main functions of MECs. Prolactin ${ }^{61}$, TTOR $^{62}$ and insulin pathways ${ }^{63}$ with a protein count of 57, 114 and 112 respectively, were found in the current dataset. These pathways were previously reported to be involved in the differentiation of MECs. The existence of molecular machinery necessary for differentiation in BuMECs demonstrates the capability of actively proliferating cells to differentiate under the appropriate stimulus. The diverse nature of growth control mechanisms in BuMECs suggests the requirement for complex cellular decisions during various developmental stages of the mammary gland. 
Also, the present study reports the presence of endopin, $\mathrm{Ca}^{2+}$ dependent activator protein (CAPS2) and Sec 1 family domain containing 1 (SCFD1) in BuMECs. These proteins were previously reported to be associated with secretory functions. Endopin 2 is a serpin and is present in the secretory vesicles ${ }^{64,65}$. CAPS2 facilitates the release of brain-derived neurotrophic factor and neurotrophin from cerebellar granule cells ${ }^{66}$. SCDF1 helps in the release of ECM proteins, which play an essential role in the differentiation of chondrocytes ${ }^{67}$. Further investigation is needed to study the role of these proteins in the BuMECs.

Three variants of aldehyde oxidase (AOX) including 1, 3L1 and 4 were identified in the actively proliferating BuMECs. AOX is a member of the molybdo-flavoenzyme family, which varies in its number and isoforms among mammals such that human express only AOX1, whereas AOX1, AOX3L1 and AOX4 are expressed in cows ${ }^{68}$. The functional significance of these enzymes in MECs is not clear. However, they have been reported to play a role in the clearance of drugs containing aldehydes and $\mathrm{N}$-containing heterocyclic fragments in liver ${ }^{69}$.

Interestingly, we identified 111 proteins in the BuMECs proteome dataset, which were associated with the oxytocin signalling pathway. Oxytocin signalling is usually present in the myoepithelial cells to facilitate the ejection of milk from the alveoli ${ }^{70}$. In contrast, Lolliver et al. ${ }^{71}$, reported the presence of oxytocin receptors in ductal MECs in the rabbit mammary gland. The protein kinase $\mathrm{C}(\mathrm{PKC})$ identified in our study is a central molecule of the oxytocin signalling. It stimulates the expression of PGF $2 \alpha$ through cyclooxygenase $2(\mathrm{COX} 2)^{72}$. Local prostaglandin may affect the blood flow, thereby regulating milk production ${ }^{73}$. Presence of proteins associated with oxytocin pathway in BuMECs, suggests that alveolar epithelial cells have oxytocin responsive mechanisms in milk ejection.

There were 85 proteins common to all fractions of BuMECs. The proteins isolated from various sub-cellular fractions were highly enriched fractions that may have resulted in carryover contamination among the fractions, although some of the common proteins may be ubiquitous. Notable among the proteins found common in five fractions were proliferation associated protein $2 \mathrm{G} 4$ (PA2G4) or ErbB3 binding protein-1 (Ebp1), serotransferrin, heat shock protein 10 (HSP10), bromodomain-containing protein 4 (BRD4), and microtubule actin crosslinking factor 1 (MACF1). PA2G4 belongs to a family of DNA/RNA binding proteins which plays an important role in cell growth, apoptosis and differentiation ${ }^{74-76}$. Serotransferrin is a glycoprotein, which is previously reported to have a role in cellular iron transport ${ }^{77}$, growth, differentiation and bacteriostatic effect ${ }^{78}$. HSP10 is a mitochondrial protein which along with HSP60 forms a complex and mainly function in the folding of proteins ${ }^{79}$. HSP 10 is reported to act through the RAF pathway and enhances the survival rate of brain cells ${ }^{80}$. BRD4 is a histone binding protein, which plays an essential role in the proliferation of the various cell types, such as squamous epithelial cells $^{81}$, macrophages ${ }^{82}$, and cerebellar granule cell progenitors ${ }^{83}$. In the brain, it acts through sonic hedgehog $(\mathrm{SHH})$ signalling pathway and regulates the levels of effector molecules, including transcription activators- Gli1 and Gli2 $2^{83,84}$. MACF1 is an actin-binding protein ${ }^{85}$, which is reported to play an essential role in the proliferation of neuronal progenitors through WNT/ $\beta$-catenin and GSK-3 signalling pathways, during development of the nervous system ${ }^{86}$.

A significant number (372) of uncharacterized proteins were found in the BuMECs proteome dataset. They may represent genes encoding small proteins or novel unannotated genes which require further investigations. These proteins/peptides in BuMECs may have species-specific functions in the mammary gland. The data generated in our study can be used for proteogenomic analysis to identify the non-annotated proteins for their significance in mammary gland biology.

Using an in-depth analysis of the BuMECs proteomics, we identified a large number of DEPs between cell fractions. This information will help to understand the role of identified proteins in mammary gland development and lactation biology. Also, it will serve as a dataset for proteome based genome annotations in buffalo species. The present BuMEC proteome dataset can be used as a reference dataset for parallel comparative studies in other species.

\section{Materials and Methods}

Cell culture of actively proliferating BuMECs and protein isolation from SCFs. The spontaneously immortalized BuMEC line developed in our lab ${ }^{5}$ was used in the present study. Cells at $25^{\text {th }}$ passage were cultivated in Dulbecco's modified Eagle's medium (DMEM) (Sigma, US) supplemented with 10\% fetal bovine serum (FBS) (Gibco, Thermo Fischer Scientific, US), $10 \mathrm{ng} / \mathrm{ml}$ EGF (Sigma, US), $200 \mathrm{mM}$ glutamine (Sigma, US), $1 \mu \mathrm{g} /$ $\mathrm{ml}$ hydrocortisone (Sigma, US) and $5 \mu \mathrm{g} / \mathrm{ml}$ bovine insulin (Sigma, US), at $37^{\circ} \mathrm{C}$ in a humidified atmosphere of $5 \% \mathrm{CO}_{2}$. The cells were grown in T-75 polystyrene flasks (Nunc, Denmark) to $70-75 \%$ confluency (Fig. 5). The protein was isolated from the sub-confluent cultures $(n=3)$ using Proteo Extract Sub-cellular Proteome Extraction Kit (S-PEK, Calbiochem) as per the manufacturer's protocol. The protein from four SCFs which included SCF-I (Cytosolic proteins), SCF-II (Membrane and Membranous Organelle's proteins), SCF-III (Nuclear proteins) and SCF-IV (Cytoskeletal proteins) was extracted separately at $4{ }^{\circ} \mathrm{C}$ (Fig. 6a). Briefly, the three independent cultures of actively proliferating BuMECs of the same passage were grown $(n=3)$, and subcellular protein fractions were isolated from these three independent cultures separately. The equal amount of protein from each independent culture was pooled together to generate a single pool for each subcellular protein fraction ${ }^{87}$. The pooled protein fraction for each SCF was then fractionated using ultra-high-performance liquid chromatography (U-HPLC), and processed for deep proteome analysis.

Clean up of isolated sub-cellular protein fractions and protein estimation. Interfering substances such as detergents, salts, lipids, and nucleic acids were removed from the four protein preparations, which included proteins from SCF-1, -II, -III and -IV, using a 2D-clean up kit (GE Healthcare, US). After cleanup, the proteins were visible as the white pellet, which was then dissolved in $2 \%$ diethylamine (DEA). The protein concentration of each sample was estimated using a 2D-Quant kit (GE Healthcare, US) as per the manufacturer's instructions. 


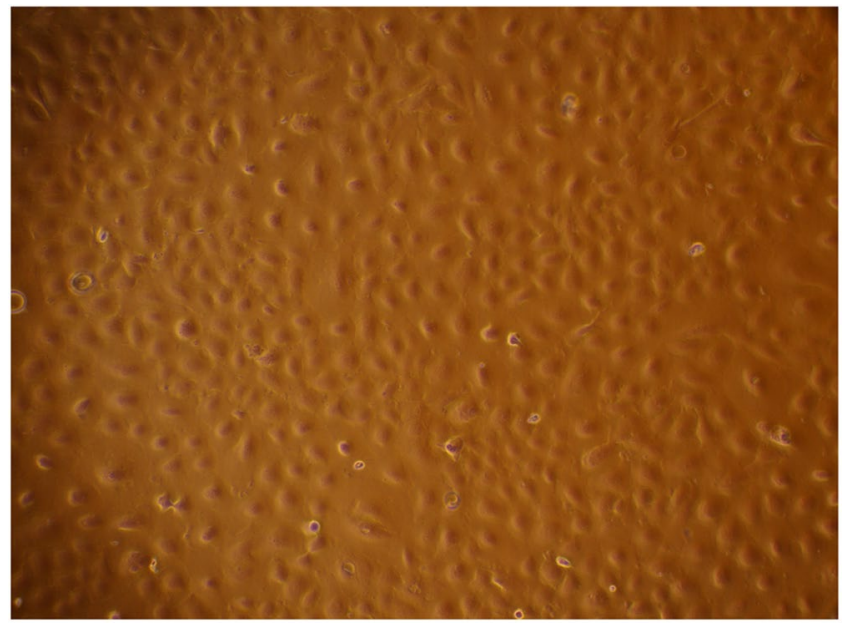

Figure 5. Phase-contrast microscopic image $(100 \times)$ (Nikon Ti Eclipse, Nikon, Japan) of actively proliferating buffalo mammary epithelial cells (BuMECs). The BuMECs were grown in DMEM supplemented with $10 \%$ FBS, $10 \mathrm{ng} / \mathrm{ml} \mathrm{EGF}, 200 \mathrm{mM}$ glutamine, $1 \mathrm{ug} / \mathrm{ml}$ hydrocortisone and $5 \mathrm{ug} / \mathrm{ml}$ Bovine insulin at $37^{\circ} \mathrm{C}$ in a humidified atmosphere of $5 \% \mathrm{CO}_{2}$. The BuMECs exhibit cobblestone appearance typical of epithelial morphology.

$$
\mathbf{a}
$$

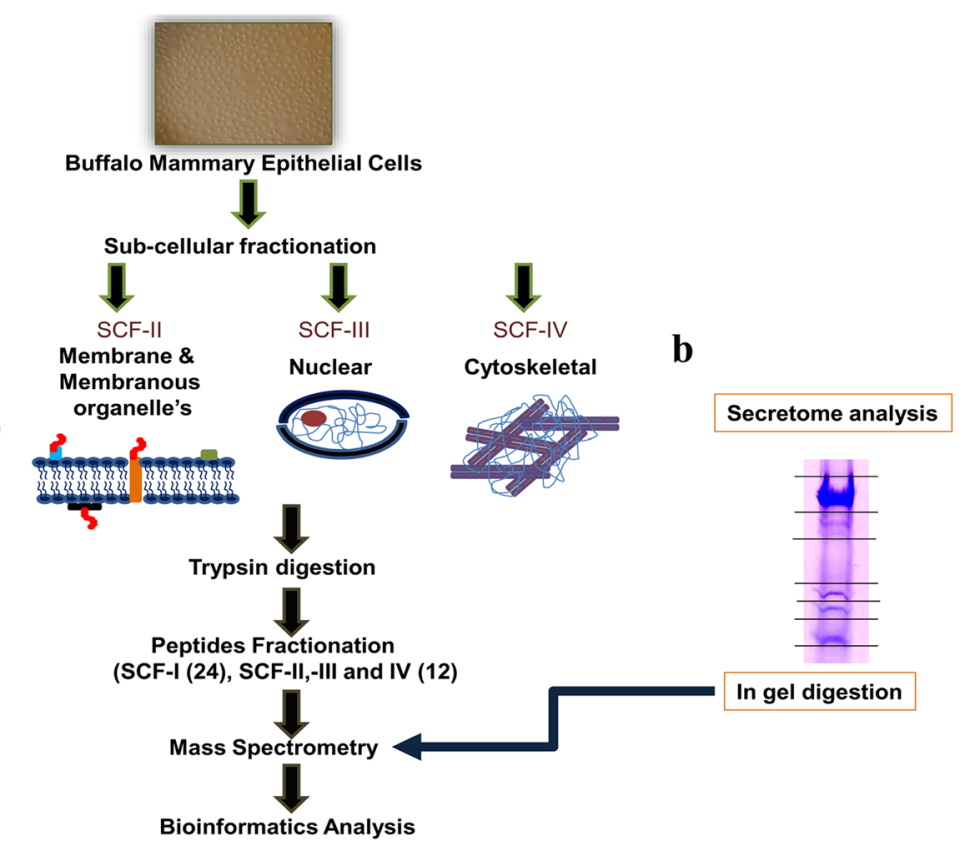

Figure 6. Schematic overview of the analytical workflow. (a) Proteomics workflow for sub-cellular protein isolation and MS analysis. The protein from four sub-cellular fractions (SCF-I-IV) was extracted separately from buffalo mammary epithelial cells (BuMECs) using sub-cellular protein extraction kit (SPEK) at $4{ }^{\circ} \mathrm{C}$. The extracted proteins were digested and fractionated using Ultra-High-Performance Liquid Chromatography (U-HPLC) into 24 and 12 individual fractions for SCF-I and SCF (-II-III- and -IV) respectively. Each resultant peptide fraction was analyzed using the nanoLC-MS/MS. The raw data (.mgf files) were analyzed using ProteinScape Software. The biological significance of identified proteins was studied using various Bioinformatics tools. (b) Workflow for secretome analysis. The protein prepared from the conditioned media was run into the SDS-PAGE (Full-length SDS-Gel image for conditioned media is given as Supplementary Fig. S1), and protein bands were cut into eight gel sections. Each gel section was digested using the In-gel digestion approach and was further analyzed using the nanoLC-MS/MS. Global cellular and secretome analysis identified a total of 12,609 proteins.

Cell culture and isolation of proteins from conditioned media for secretome analysis. The BuMECs $\left(25^{\text {th }}\right.$ passage $)$ were maintained as described above and grown up to $70-75 \%$ confluency. Subsequently, the media was aspirated, and the cells were rinsed twice with 1X DPBS (Sigma, US) and once with DMEM/F-12, to remove the serum proteins efficiently. The rinsed cells were grown further for $28 \mathrm{~h}$ in serum-free DMEM/F-12 
media supplemented with $10 \mathrm{ng} / \mathrm{ml}$ EGF, $200 \mathrm{mM}$ glutamine, $1 \mu \mathrm{g} / \mathrm{ml}$ hydrocortisone and $5 \mu \mathrm{g} / \mathrm{ml}$ bovine insulin. Conditioned media were collected from each sample $(n=3)$, and filtered using a syringe filter with a $0.22 \mu \mathrm{m}$ pore size. The filtered conditioned media was then centrifuged at $1,500 \mathrm{RPM}, 4^{\circ} \mathrm{C}$ for $10 \mathrm{~min}$, to remove the cell debris. The supernatant was collected and concentrated using a $3 \mathrm{kDa}$ molecular weight cutoff centricon (Millipore, Sigma, US) at $4,000 \mathrm{~g}, 4^{\circ} \mathrm{C}$. The protease inhibitor (Roche, Switzerland) was added into the protein prepared from the conditioned media and was stored at $-80^{\circ} \mathrm{C}$ until further processing for MS

In-solution tryptic digestion of isolated sub-cellular protein fractions. For In-solution digestion, $365 \mu \mathrm{g}, 200 \mu \mathrm{g}, 165 \mu \mathrm{g}$ and $120 \mu \mathrm{g}$ of the protein from four SCFs (SCF-I, SCF-II, SCF-III and SCF-IV) respectively, was lyophilized and reconstituted in $100 \mathrm{mM}$ triethylammonium bicarbonate (TEAB) buffer (Sigma, US). Protein from each SCF was processed separately for trypsin digestion. Briefly, $45 \mathrm{mM}$ dithiothreitol (DTT) (Sigma, US) was added into the protein sample, which was then incubated at $60^{\circ} \mathrm{C}$ for $1 \mathrm{~h}$ to reduce the disulfide bonds. Subsequently, $10 \mathrm{mM}$ iodoacetamide (IAA) (Sigma, US) was added and the protein sample was incubated in the dark for $30 \mathrm{~min}$ to alkylate the cysteine residues. The protein was then digested into peptides by overnight treatment with trypsin (Promega, US) $(1: 20)$ at $37^{\circ} \mathrm{C}$. The reaction was subsequently stopped with $10 \%$ trifluoroacetic acid (TFA) (Sigma, US), followed which the peptides were vacuum dried and stored at $-80^{\circ} \mathrm{C}$ until further use.

Fractionation of digested sub-cellular proteins using U-HPLC. The fractionation of the digested peptides from each SCF was performed using U-HPLC ${ }^{88}$. Briefly, the digested protein sample was loaded onto the C18 column $(4.6 \times 250 \mathrm{~mm}, 5 \mu \mathrm{m}$, Grace, US) on a Dionex, Quaternary U-HPLC system (Ultimate 3000 , Thermo, US) with a UV detection at $214 \mathrm{~nm}$. Two HPLC solvents used were, Solvent A which was $10 \mathrm{mM} \mathrm{TEAB}$ and Solvent B which was $10 \mathrm{mM}$ TEAB in $90 \%$ acetonitrile (ACN) (Sigma, US). The peptides were fractionated at $25^{\circ} \mathrm{C}$ with a flow rate of $1 \mathrm{ml} / \mathrm{min}$ and continuous gradient elution $(5-100 \% \mathrm{ACN}$ ) from the column over $81 \mathrm{~min}$. The linear gradient was set up as follows: 0 to $2 \%$ B for $5 \mathrm{~min}, 2$ to $60 \% \mathrm{~B}$ for $60 \mathrm{~min}, 60$ to $100 \% \mathrm{~B}$ for $10 \mathrm{~min}$, held the same gradient for next $1 \mathrm{~min}$ followed by $2 \%$ B for $5 \mathrm{~min}$. Digested protein sample from each fraction was run and fractionated separately using the U-HPLC system. A total of 96 time-based fractions were collected and further pooled into 24 and 12 individual fractions for SCF-1 and SCFs (-II, -III and -IV) respectively. The pooling of peptide fractions was done by mixing most hydrophobic ones with most hydrophilic based on concatenation approach. The pooled peptide fractions were then lyophilized, acidified in $0.1 \%$ formic acid (FA) (Sigma, US) and desalted using C18 Zip tips (Millipore, US). The desalted peptides were lyophilized and stored at $-80^{\circ} \mathrm{C}$ before subjecting to nanoLC-MS/MS analysis.

One Dimensional Gel Electrophoresis (1D-GE) and In-gel tryptic digestion of the protein sample prepared from conditioned media. The concentrated conditioned media was quantified using the 2-D Quant kit as per the manufacturer's instructions. $25 \mu \mathrm{g}$ of protein from conditioned media was subjected to $12 \%$ SDS-PAGE $(10 \times 10.5 \mathrm{~cm})$ in a Mini VE complete gel electrophoresis system (GE Healthcare, Sigma, US). The protein bands were visualized by Coomassie brilliant blue (R-350) staining (Fig. S1). The image was obtained using EPSON Scanner (Image ScGE Healthcare, Bio-Science, US). The stained protein bands were then cut into eight equal pieces, and In-gel digestion was performed as reported previously ${ }^{89}$ (Fig. 6b). Briefly, the gel bands were washed with Milli-Q water and destained using $40 \mathrm{mM}$ ammonium bicarbonate $\left(\mathrm{NH}_{4} \mathrm{HCO}_{3}\right)$ in $50 \% \mathrm{ACN}$ at a ratio of $1: 1(\mathrm{v} / \mathrm{v})$ followed by rehydration with $100 \% \mathrm{ACN}$ for $10 \mathrm{~min}$. The protein in destained bands was reduced by $10 \mathrm{mM}$ DTT followed by alkylation using $55 \mathrm{mM}$ IAA. The overnight digestion of reduced and alkylated protein was done with trypsin $(1: 20)$ at $37^{\circ} \mathrm{C}$. The reaction was then stopped with $5 \% \mathrm{TFA}$, and the digested protein samples were lyophilized. The lyophilized peptides were acidified in $0.1 \%$ FA and desalted using C18 Zip-tips following the manufacturer's instructions. The peptides were stored at $-80^{\circ} \mathrm{C}$ until nanoLC-MS/MS analysis.

NanoLC-MS/MS analysis. The fractionated peptides were reconstituted in $0.1 \%$ FA, followed which the proteins were identified using captive spray-Maxis-HD qTOF (Bruker Daltonics, Germany) MS with high mass accuracy and sensitivity. The peptides were initially enriched on a nano-trap column $(\mathrm{C} 18,2 \mathrm{~cm}, 5 \mu, 100 \AA$, Agilent), followed by elution on to analytical column $(15 \mathrm{~cm}, 3 \mu, 100 \AA$, Agilent). The peptides were sprayed using Nano electrospray emitter tip of $10 \mu \mathrm{m}$ (Bruker, Germany) using $0.1 \%$ FA in water as solvent A, and $0.1 \%$ FA in $\mathrm{ACN}$ as solvent $\mathrm{B}$. The peptides were loaded onto the trap column using $97 \%$ solvent $\mathrm{A}$, followed by resolution on the analytical column using a linear gradient of 5-30\% solvent B for $70 \mathrm{~min}$ at a constant flow rate of $400 \mathrm{nl} / \mathrm{min}$. The data were acquired in data-dependent acquisition mode subjecting the six most intense ions in each survey scan to MS/MS analysis within a $\mathrm{m} / \mathrm{z}$ range of 400-2200. The collision-induced dissociation (CID) method was used for precursor fragmentation, and the precursor ions selected for MS/MS fragmentation were excluded after every three spectra. The absolute threshold for precursor ions per 1000 summations was 1200 counts $^{88,90}$.

Database searching and protein identification. Peak lists were generated by qTOF control (version 24.8) using the Hystar post-processing program to automatically subtract baseline, smoothen peaks and to generate centroid data. The data so generated was searched against the Bos taurus database for identification and quantification of proteins using Mascot (2.4.1 Matrix Science, UK) search engine in ProteinScape software 3.2 (Buker Daltonics, Germany) ${ }^{88,90,91}$. Proteins were identified by correlation of mass spectra to entries in the Bos taurus database, UniProt (https://www.uniprot.org/) ${ }^{92}$. Mascot MS/MS ion search criteria used were as follows: taxonomy-other Mammalia, specific digestion with trypsin, allowing up to one missed cleavage, MS/MS tolerance of $0.05 \mathrm{Da}$ and peptide tolerance of $50 \mathrm{ppm}$. The Carbamidomethylation of cysteine was searched as a fixed modification, whereas Carbamyl, Carboxymethyl, Oxidation, and Phospho were set as the variable modifications. The "ion score cutoff" was manually set to 15 , thereby eliminating the lowest quality matches. To eliminate the false positives, a 1\% FDR was applied at both protein and peptide levels. The raw MS data and the ProteinScape 
output has been deposited to the ProteomeXchange Consortium (http://proteomecentral.proteomexchange.org) via the PRotemics IDEntifications database (PRIDE) ${ }^{93}$ partner repository with the dataset identifier PXD013707. The secretory proteins in the conditioned media were identified using the web-based Bioinformatics tool, SignalP 5.0 (www.cbs.dtu.dk/services/SignalP/) ${ }^{94}$.

GO and pathway enrichment analysis of the identified proteins. To evaluate the significance of proteins identified in the BuMECs proteome dataset, GO analysis was done using PANTHER. The proteins were classified into three categories which included BP, MF and CC. To identify the pathways associated with the identified proteins, the KEGG and DAVID, version 6.7 (https://david.ncifcrf.gov) analysis were performed. Fisher exact corrected $p$-values $\leq 0.05$ were considered significant.

Construction of the PPI network and core protein sub-network analysis. The PPI network analysis was performed using STRING ${ }^{15}$ and Cytoscape. The proteins were mapped to the STRING database, and PPIs with the highest confidence score $(\geq 0.9)$ were selected. These PPIs were then analyzed using the Cytoscape. The densely connected PPIs or modules were screened out from the main PPI network with the degree cutoff $=2$, node score cutoff $=0.2, \mathrm{k}$-core $=2$ and $\max$ depth $=100$ using MCODE, a Cytoscape plug-in ${ }^{16}$. To better understand the functions of proteins in modules, pathway enrichment analysis was performed using the Reactome Functional Interaction (FI) plug-in 6.1.0.

Consent for publication. The authors gave their consent for publication of the research results.

\section{Data availability}

RAW MS data have been deposited to the ProteomeXchange Consortium via the PRIDE partner repository with the dataset identifier PXD013707. RAW data were analyzed using the ProteinScape Software, and the output has also been uploaded to the ProteomeXchange Consortium under the same identifier.

Received: 30 July 2019; Accepted: 25 February 2020;

Published online: 16 March 2020

\section{References}

1. Robinson, G. W. Cooperation of signalling pathways in embryonic mammary gland development. Nature Reviews Genetics $\mathbf{8}$, 963-972 (2007)

2. Khokha, R. \& Werb, Z. Mammary gland reprogramming: metalloproteinases couple form with function. Cold Spring Harbor Perspectives in Biology 3, a004333, https://doi.org/10.1101/cshperspect.a004333 (2011).

3. Siddiky, M. N. A. \& Faruque, M. O. Buffaloes for dairying in South Asia: potential, challenges and way forward. SAARC. Journal of Agriculture 15, 227-239 (2017).

4. Hamid, M. A., Ahmed, S., Rahman, M. A. \& Hossain, K. M. Status of buffalo production in Bangladesh compared to SAARC countries. Asian Journal of Animal Sciences 10, 313-329 (2016).

5. Anand, V. et al. Establishment and characterization of a buffalo (Bubalus bubalis) mammary epithelial cell line. PLoS ONE 7, e40469, https://doi.org/10.1371/journal.pone.0040469 (2012).

6. Janjanam, J. et al. Proteome analysis of functionally differentiated bovine (Bos indicus) mammary epithelial cells isolated from milk. Proteomics 13, 3189-3204 (2013).

7. Sandhu, C., Connor, M., Kislinger, T., Slingerland, J. \& Emili, A. Global protein shotgun expression profiling of proliferating MCF-7 breast cancer cells. Journal of Proteome Research 4, 674-689 (2005).

8. Geiger, T., Wehner, A., Schaab, C., Cox, J. \& Mann, M. Comparative proteomic analysis of eleven common cell lines reveals ubiquitous but varying expression of most proteins. Molecular \& Cellular Proteomics 11, https://doi.org/10.1074/mcp.M111.014050 (2012).

9. Gholami, A. M. et al. Global proteome analysis of the NCI-60 cell line panel. Cell Reports 4, 609-620 (2013).

10. Geiger, T. et al. Initial quantitative proteomic map of twenty-eight mouse tissues using the SILAC mouse. Molecular \& Cellular Proteomics 12, 1709-1722 (2013).

11. Fagerberg, L. et al. Mapping the sub-cellular protein distribution in three human cell lines. Journal of Proteome Research 10, 3766-3777 (2011).

12. Mi, H. et al. PANTHER version 11: expanded annotation data from Gene Ontology and Reactome pathways, and data analysis tool enhancements. Nucleic Acids Research 45, D183-D189 (2016).

13. Gao, J. et al. Metscape: a Cytoscape plug-in for visualizing and interpreting metabolomic data in the context of human metabolic networks. Bioinformatics 26, 971-973 (2010).

14. Sevimoglu, T. \& Arga, K. Y. The role of protein interaction networks in systems biomedicine. Computational and Structural Biotechnology Journal 11, 22-27 (2014).

15. Szklarczyk, D. et al. STRING v10: protein-protein interaction networks, integrated over the tree of life. Nucleic Acids Research 43, D447-D452 (2015).

16. Bader, G. D. \& Hogue, C. W. V. An automated method for finding molecular complexes in large protein interaction networks. BMC Bioinformatics 4, 2, https://doi.org/10.1186/1471-2105-4-2 (2003).

17. Nagamura-Inoue, T., Tamura, T. \& Ozato, K. Transcription factors that regulate growth and differentiation of myeloid cells. International Reviews of Immunology 20, 83-105 (2001).

18. Huang, D. W. et al. The DAVID Gene Functional Classification Tool: a novel biological module-centric algorithm to functionally analyze large gene lists. Genome Biology 8, R183, https://doi.org/10.1186/gb-2007-8-9-r183 (2007).

19. Bardou, P., Mariette, J., Escudié, F., Djemiel, C. \& Klopp, C. Jvenn: an interactive Venn diagram viewer. BMC Bioinformatics 15, 293, https://doi.org/10.1186/1471-2105-15-293 (2014).

20. Boutinaud, M., Lollivier, V., Finot, L., Bruckmaier, R. M. \& Lacasse, P. Mammary cell activity and turnover in dairy cows treated with the prolactin-release inhibitor quinagolide and milked once daily. Journal of Dairy Science 95, 177-187 (2012).

21. Jena, M. K. et al. DIGE based proteome analysis of mammary gland tissue in water buffalo (Bubalus bubalis): lactating vis-a-vis heifer. Journal of Proteomics 119, 100-111 (2015).

22. Janjanam, J. et al. Comparative 2D-DIGE proteomic analysis of bovine mammary epithelial cells during lactation reveals protein signatures for lactation persistency and milk yield. PLoS ONE 9, e102515, https://doi.org/10.1371/journal.pone.0102515 (2014).

23. Murgiano, L. et al. Comparison of milk fat globule membrane (MFGM) proteins of Chianina and Holstein cattle breed milk samples through proteomics methods. Nutrients 1, 302-315 (2009). 
24. Ma, Y., Zhang, L., Wu, Y. \& Zhou, P. Changes in milk fat globule membrane proteome after pasteurization in human, bovine and caprine species. Food Chemistry 279, 209-215 (2019).

25. Li, W. et al. Quantitative proteomic analysis of milk fat globule membrane (MFGM) proteins from donkey colostrum and mature milk. Food \& Function 10, 4256-4268 (2019).

26. Davies, C. R. et al. Proteomic analysis of the mouse mammary gland is a powerful tool to identify novel proteins that are differentially expressed during mammary development. Proteomics 6, 5694-5704 (2006).

27. Moreira, J. M. et al. Tissue proteomics of the human mammary gland: towards an abridged definition of the molecular phenotypes underlying epithelial normalcy. Molecular Oncology 4, 539-561 (2010).

28. Russo, J. \& Russo, I. H. Development of the human breast. Maturitas 49, 2-15 (2004).

29. Tucker, H. A. Factors affecting mammary gland cell numbers. Journal of Dairy Science 52, 720-729 (1969).

30. Rauner, G., Leviav, A., Mavor, E. \& Barash, I. Development of foreign mammary epithelial morphology in the stroma of immunodeficient mice. PLoS ONE 8, e68637, https://doi.org/10.1371/journal.pone.0068637 (2013).

31. Li, G. et al. abLIM1 constructs non-erythroid cortical actin networks to prevent mechanical tension-induced blebbing. Cell Discovery 4, 42, https://doi.org/10.1038/s41421-018-0040-3 (2018).

32. Bissonnette, N., Dudemaine, P. L., Thibault, C. \& Robitaille, G. Proteomic analysis and immunodetection of the bovine milk osteopontin isoforms. Journal of Dairy Science 95, 567-579 (2012).

33. Espiritu, E. B. et al. The Lhx1-Ldb1 complex interacts with Furry to regulate microRNA expression during pronephric kidney development. Scientific Reports 8, 16029, https://doi.org/10.1038/s41598-018-34038-x (2018).

34. Byun et al. Fryl deficiency is associated with defective kidney development and function in mice. Experimental Biology and Medicine 243, 408-417 (2018).

35. Weng, Y. R., Cui, Y. \& Fang, J. Y. Biological functions of cytokeratin 18 in cancer. Molecular Cancer Research 10, 485-493 (2012).

36. Smalley, M. J. et al. Differentiation of separated mouse mammary luminal epithelial and myoepithelial cells cultured on EHS matrix analyzed by indirect immunofluorescence of cytoskeletal antigens. Journal of Histochemistry « Cytochemistry 47, 1513-1524 (1999).

37. Hu, W. et al. Stem cell lineage hierarchy by keratin profiling in normal human prostate epithelial cells and prostate cancer. Tumor Biology, https://doi.org/10.1158/1538-7445.AM2018-3063 (2018).

38. Trask, D. K. et al. Keratins as markers that distinguish normal and tumor-derived mammary epithelial cells. Proceedings of the National Academy of Sciences 87, 2319-2323 (1990).

39. Iyer, S. V. et al. Understanding the role of keratins 8 and 18 in neoplastic potential of breast cancer derived cell lines. PLoS ONE 8 , e53532, https://doi.org/10.1371/journal.pone.0053532 (2013).

40. Lu, B. et al. Extracellular calcium controls background current and neuronal excitability via an UNC79-UNC80-NALCN cation channel complex. Neuron 68, 488-499 (2010).

41. Ren, D. Sodium leak channels in neuronal excitability and rhythmic behaviors. Neuron 72, 899-911 (2011).

42. Laforenza, U. et al. Aquaporin-6 is expressed along the rat gastrointestinal tract and upregulated by feeding in the small intestine. BMC Physiology 9, 18, https://doi.org/10.1186/1472-6793-9-18 (2009).

43. He, J. \& Yang, B. Aquaporins in renal diseases. International Journal of Molecular Sciences 20, 366, https://doi.org/10.3390/ ijms20020366 (2019).

44. Mobasheri, A. et al. Cellular localization of aquaporins along the secretory pathway of the lactating bovine mammary gland: an immunohistochemical study. Acta Histochemica 113, 137-149 (2011).

45. Galán-Cobo, A., Ramírez-Lorca, R. \& Echevarría, M. Role of aquaporins in cell proliferation: What else beyond water permeability? Channels 10, 185-201 (2016).

46. Xia, J. et al. Ion channels or aquaporins as novel molecular targets in gastric cancer. Molecular Cancer 16, 54, https://doi.org/10.1186/ s12943-017-0622-y (2017).

47. Vasiliou, V., Vasiliou, K. \& Nebert, D. W. Human ATP-binding cassette (ABC) transporter family. Human Genomics 3, 281-290 (2009).

48. Langmann, T. et al. Real-time reverse transcription-PCR expression profiling of the complete human ATP-binding cassette transporter superfamily in various tissues. Clinical Chemistry 49, 230-238 (2003).

49. Farke, C., Meyer, H. H. D., Bruckmaier, R. M. \& Albrecht, C. Differential expression of ABC transporters and their regulatory genes during lactation and dry period in bovine mammary tissue. Journal of Dairy Research 75, 406-414 (2008).

50. Shen, Y. et al. TSPY1 suppresses USP7-mediated p53 function and promotes spermatogonial proliferation. Cell Death \& Disease 9 , 542, https://doi.org/10.1038/s41419-018-0589-7 (2018).

51. Duffy, M. J. et al. The ADAMs family of proteases: new biomarkers and therapeutic targets for cancer? Clinical Proteomics 8, 9, https://doi.org/10.1186/1559-0275-8-9 (2011).

52. McGowan, P. M. et al. ADAM-17 expression in breast cancer correlates with variables of tumor progression. Clinical Cancer Research 13, 2335-2343 (2007).

53. Groot, A. J. \& Vooijs, M. A. The role of Adams in Notch signaling. Notch Signaling in Embryology and Cancer 727, 15-36 (2012).

54. Edwards, D. R., Handsley, M. M. \& Pennington, C. J. The ADAM metalloproteinases. Molecular Aspects of Medicine 29, 258-289 (2008).

55. Castrillon, D. H., Quade, B. J., Wang, T. Y., Quigley, C. \& Crum, C. P. The human VASA gene is specifically expressed in the germ cell lineage. Proceedings of the National Academy of Sciences 97, 9585-9590 (2000).

56. Lee, K. B. et al. Functional role of the bovine oocyte-specific protein JY-1 in meiotic maturation, cumulus expansion, and subsequent embryonic development. Biology of Reproduction 90, 69, https://doi.org/10.1095/biolreprod.113.115071 (2014).

57. Capuco, A. V. et al. Lactation persistency: insights from mammary cell proliferation studies. Journal of Animal Science 81, 18-31 (2003).

58. Ma, Z., Liu, X., Zhang, Q., Yu, Z. \& Gao, D. Carvedilol suppresses malignant proliferation of mammary epithelial cells through inhibition of the ROS-mediated PI3K/AKT signalling pathway. Oncology Reports 41, 811-818 (2019).

59. Jankiewicz, M., Groner, B. \& Desrivières, S. Mammalian target of rapamycin regulates the growth of mammary epithelial cells through the inhibitor of deoxyribonucleic acid binding Id1 and their functional differentiation through Id2. Molecular Endocrinology 20, 2369-2381 (2006)

60. Kim, N. S. et al. Receptor activator of NF-kB ligand regulates the proliferation of mammary epithelial cells via Id2. Molecular and Cellular Biology 26, 1002-1013 (2006).

61. Liu, F. et al. Prolactin/Jak2 directs apical/basal polarization and luminal linage maturation of mammary epithelial cells through regulation of the Erk1/2 pathway. Stem Cell Research 15, 376-383 (2015).

62. Lu, L. M., Li, Q. Z., Huang, J. G. \& Gao, X. J. Proteomic and functional analyzes reveal MAPK1 regulates milk protein synthesis. Molecules 18, 263-275 (2012).

63. Bionaz, M. \& Loor, J. J. Gene networks driving bovine mammary protein synthesis during the lactation cycle. Bioinformatics and Biology Insights 5, 83-98 (2011).

64. Hwang, S. R. et al. The novel serpin endopin 2 demonstrates cross-class inhibition of papain and elastase: localization of endopin 2 to regulated secretory vesicles of neuroendocrine chromaffin cells. Biochemistry 41, 10397-10405 (2002).

65. Hook, V. Y. \& Hwang, S. R. Novel secretory vesicle serpins, endopin 1 and endopin 2: endogenous protease inhibitors with distinct target protease specificities. Biological Chemistry 383, 1067-1074 (2002). 
66. Sadakata, T., Washida, M., Morita, N. \& Furuichi, T. Tissue distribution of Ca2 -dependent activator protein for secretion family members CAPS1 and CAPS2 in mice. Journal of Histochemistry \& Cytochemistry 55, 301-311 (2007).

67. Hou, N., Yang, Y., Scott, I. C. \& Lou, X. The Sec domain protein Scfd1 facilitates trafficking of ECM components during chondrogenesis. Developmental Biology 421, 8-15 (2017).

68. Garattini, E., Fratelli, M. \& Terao, M. Mammalian aldehyde oxidases: genetics, evolution and biochemistry. Cellular and Molecular Life Sciences 65, 1019-1048 (2008).

69. Montefiori, M., Jørgensen, F. S. \& Olsen, L. Aldehyde oxidase: reaction mechanism and prediction of site of metabolism. ACS Omega 2, 4237-4244 (2017).

70. Haaksma, C. J., Schwartz, R. J. \& Tomasek, J. J. Myoepithelial cell contraction and milk ejection are impaired in mammary glands of mice lacking smooth muscle alpha-actin. Biology of Reproduction 85, 13-21 (2011).

71. Lollivier, V. et al. Oxytocin stimulates secretory processes in lactating rabbit mammary epithelial cells. The Journal of Physiology 570, $125-140$ (2006)

72. Wouters, E., Hudson, C. A., McArdle, C. A. \& Bernal, A. L. Central role for protein kinase C in oxytocin and epidermal growth factor stimulated cyclooxygenase 2 expression in human myometrial cells. BMC Research Notes 7, 357, https://doi.org/10.1186/1756-05007-357 (2014).

73. Kimura, T. et al. Expression and immunolocalization of the oxytocin receptor in human lactating and non-lactating mammary glands. Human Reproduction 13, 2645-2653 (1998).

74. Zhang, Y. et al. Alterations in cell growth and signaling in ErbB3 binding protein-1 (Ebp1) deficient mice. BMC Cell Biology 9, 69, https://doi.org/10.1186/1471-2121-9-69 (2008).

75. Figeac, N., Serralbo, O., Marcelle, C. \& Zammit, P. S. ErbB3 binding protein-1 (Ebp1) controls proliferation and myogenic differentiation of muscle stem cells. Developmental Biology 386, 135-151 (2014).

76. Zhu, L., Lee, Y., Ta, L. \& Mitchell, B. S. Expression and role of the ErbB3-binding protein 1 in acute myelogenous leukemic cells. Clinical Cancer Research 22, 3320-3327 (2016).

77. Al-wajeeh, A. S. et al. Identification of Glycobiomarker Candidates for Breast Cancer Using LTQ-Orbitrap Fusion Technique. International Journal of Pharmacology 13, 425-437 (2017).

78. Bartnikas, T. B. Known and potential roles of transferrin in iron biology. Biometals 25, 677-686 (2012)

79. Jia, H. et al. Heat shock protein 10 (Hsp10) in immune-related diseases: one coin, two sides. International Journal of Biochemistry and Molecular Biology 2, 47-57 (2011).

80. Fan, W. et al. Elevated expression of HSP10 protein inhibits apoptosis and associates with poor prognosis of astrocytoma. PLoS ONE 12, e0185563, https://doi.org/10.1371/journal.pone.0185563(2017).

81. Xiang, T. et al. Bromodomain protein BRD4 promotes cell proliferation in skin squamous cell carcinoma. Cellular Signalling $\mathbf{4 2}$, 106-113 (2018)

82. Dey, A. et al. BRD4 directs hematopoietic stem cell development and modulates macrophage inflammatory responses. The EMBO Journal 38, e100293, https://doi.org/10.15252/embj.2018100293 (2019).

83. Penas, C. et al. Time series modeling of cell cycle exit identifies Brd4 dependent regulation of cerebellar neurogenesis. Nature Communications 10, 3028, https://doi.org/10.1038/s41467-019-10799-5 (2019).

84. Ali, S. A., Niu, B., Cheah, K. S. \& Alman, B. Unique and overlapping GLI1 and GLI2 transcriptional targets in neoplastic chondrocytes. PLoS ONE 14, e0211333, https://doi.org/10.1371/journal.pone.0211333 (2019).

85. Hu, L., Huang, Z., Wu, Z., Ali, A. \& Qian, A. Mammalian Plakins, Giant Cytolinkers: Versatile Biological Functions and Roles in Cancer. International Journal of Molecular Sciences 19, 974, https://doi.org/10.3390/ijms19040974 (2018).

86. Moffat, J. J., Ka, M., Jung, E. M., Smith, A. L. \& Kim, W. Y. The role of MACF1 in nervous system development and maintenance. Seminars in Cell \& Developmental Biology 69, 9-17 (2017).

87. Stoddart, M. J., Richards, R. G. \& Alini, M. In vitro experiments with primary mammalian cells: to pool or not to pool. Eur Cell Mater 24, i-ii, https://doi.org/10.22203/eCM (2012).

88. Naru, J. et al. Proteomic analysis of differentially expressed proteins in vitreous humor of patients with retinoblastoma using iTRAQcoupled ESI-MS/MS approach. Tumor Biology 37, 13915-13926 (2016).

89. Rawat, P. et al. Identification of potential protein biomarkers for early detection of pregnancy in cow urine using 2D DIGE and label free quantitation. Clinical Proteomics 13, 15, https://doi.org/10.1186/s12014-016-9116-y (2016).

90. Bathla, S. et al. Profiling of urinary proteins in Karan Fries cows reveals more than 1550 proteins. Journal of Proteomics 127, 193-201 (2015).

91. McHugh, L. \& Arthur, J. W. Computational methods for protein identification from mass spectrometry data. PLoS Computational Biology 4, e12, https://doi.org/10.1371/journal.pcbi.0040012 (2008)

92. UniProt Consortium, UniProt: a worldwide hub of protein knowledge. Nucleic Acids Research 47, D506-D515 (2019).

93. Perez-Riverol, Y. et al. The PRIDE database and related tools and resources in 2019: improving support for quantification data. Nucleic Acids Research 47, D442-D450 (2019).

94. Lum, G. \& Min, X. J. FunSecKB: the fungal secretome knowledgebase. Database, https://doi.org/10.1093/database/bar001 (2011).

\section{Acknowledgements}

The authors are grateful to the National Agriculture Innovation Project (NAIP) (Grant Reference No. - 4136_C4), Indian Council of Agricultural Research (ICAR), Government of India (GOI) for the funding support. SJ was supported by fellowships from ICAR and University Grant Commission (UGC) for doctoral research work. SJ is also grateful to the Biotechnology Information System (BTIS), NDRI, ICAR, Karnal, India for carrying out the Bioinformatics analysis.

\section{Author contributions}

S.J. designed and performed experiments, conducted data analysis and prepared manuscript; V.A. helped in designing the experiments and in drafting the manuscript; S.K. helped in data analysis; S.B. runs the samples in MS; A.K.D. helped in designing the experiments; J.K.K. helped in data analysis; and A.K.M. supervised the project and reviewed the manuscript.

\section{Competing interests}

The authors declare no competing interests.

Additional information

Supplementary information is available for this paper at https://doi.org/10.1038/s41598-020-61521-1. 
Correspondence and requests for materials should be addressed to A.K.M.

Reprints and permissions information is available at www.nature.com/reprints.

Publisher's note Springer Nature remains neutral with regard to jurisdictional claims in published maps and institutional affiliations.

(1) Open Access This article is licensed under a Creative Commons Attribution 4.0 International License, which permits use, sharing, adaptation, distribution and reproduction in any medium or format, as long as you give appropriate credit to the original author(s) and the source, provide a link to the Creative Commons license, and indicate if changes were made. The images or other third party material in this article are included in the article's Creative Commons license, unless indicated otherwise in a credit line to the material. If material is not included in the article's Creative Commons license and your intended use is not permitted by statutory regulation or exceeds the permitted use, you will need to obtain permission directly from the copyright holder. To view a copy of this license, visit http://creativecommons.org/licenses/by/4.0/.

(C) The Author(s) 2020 